\title{
Who needs the diabetes specialist physician?
}

\author{
E. A. M. Gale
}

Published online: 29 February 2008

(C) Springer-Verlag 2008

The umbilicus serves no useful purpose other than to remind us where we came from. The sleeve of a man's jacket still carries the buttons once placed there to discourage the owner from wiping his nose. The diabetes specialist physician is seen by some health service planners in much the same light. When diabetes was rare, the specialist was badly needed. He was seen as a valued repository of unshared knowledge and took personal responsibility for a high proportion of those affected. His expertise went unchallenged, and other health professionals would seek his advice when treating a patient on insulin. The old-school diabetologist was a general physician who would, as I once did, think nothing of passing a sigmoidoscope or injecting a joint in the diabetic clinic. But times have changed, and those days are just a memory.

The most obvious change has been the increasing prevalence of diabetes. In the UK, for example, we have roughly one diabetes specialist physician for every 100,000 people, about $15 \%$ of whom will be expected to develop diabetes in the course of a normal lifespan. Since only a small minority of those affected will ever be able to consult a specialist, primary care has expanded to meet the need. General practitioners no longer fear diabetes, and (at least in the UK) are well remunerated for ticking all the treatment boxes in their patient registers. The physician, meanwhile, now works in a team and sees many of his former tasks performed, rather

\section{E. A. M. Gale}

Diabetes and Metabolism, Division of Medicine,

University of Bristol,

Bristol, UK

\section{E. A. M. Gale $(\triangle)$}

Diabetes and Metabolism, Medical School Unit,

Southmead Hospital,

Bristol BS10 5NB, UK

e-mail: Edwin.Gale@bristol.ac.uk more effectively, by others. Diabetes networks have evolved, treatment pathways have been automated, and patient care has become more streamlined, more evidence-based and more devolved. The emphasis has swung from problem management to prevention, and from the individual to the population. All this, as people tend to forget, arose on the foundation laid by generations of diabetes physicians - but have they rendered themselves obsolete in the process?

The diabetes physician has never enjoyed high standing within the hierarchy of medical specialities, for specialists still derive their status from the organ they serve. A tradition stretching back to the Middle Ages has decreed that the noble organs are situated above the diaphragm, and the greatest public respect (and private fees) are reserved for those who pronounce upon them. The sub-diaphragmatic specialities, diabetes included, are assigned to a lower caste, in keeping with the digestive and excretory functions with which they deal. Nor, in the current climate, is it easy for a diabetologist to justify his (or her) existence. Anyone can diagnose diabetes, and there are no expensive and invasive procedures that only the specialist can perform. Diabetes can be managed with negligent ease by those inclined to do so. The expertise of the physician may be wide-ranging, but he typically knows less about the workings of glucose meters and infusion pumps than his specialist nurse, less about diet than his dietitian, less about feet than the podiatrist, less about eyes, hearts, nerves, kidneys and flaccid penises than his colleagues in the corresponding specialities, and (in one or two instances known to the author) less about people than the clinic receptionist.

Nearly a century ago, a frustrated physician bemoaned the fact that a raised blood glucose level produces no pain. Life would be so much easier if it did, for affected individuals would beat a path to your door. Those who consult a venereologist have a burning desire to be there, but the 
diabetologist sees people who generally feel well. Ever ready to offer reassurance, he must first induce the anxiety that the reassurance is intended to relieve. He does this by informing people of problems they haven't got, before suggesting that they will be able to reduce the theoretical possibility of something that might not occur by abstaining from many of the simple comforts of life. Should the worst indeed happen, the diabetologist is unable to help, for his patient now needs a cardiologist, a nephrologist or an ophthalmologist. These specialities once looked to him for support, but so much of their work now relates to diabetes that they have become increasingly expert in its management. Nor will they fail to rebuke him for the fact that his patient is not in better control of his diabetes, which (to rub salt in the wounds) now improves dramatically once the patient has something to be really scared about.

Faced with this gloomy catalogue, it might be easy to conclude that the diabetologist fits the surgical definition of a physician as someone who knows everything but can do nothing. But there is worse to come, for he no longer knows everything. His opinions have come under the spotlight, for the health service apparatchik, one of an emerging breed, wishes to ensure that people with diabetes get the best available advice wherever they seek it. Simple enough, as the apparatchik will argue. You assemble the evidence, analyse it according to an agreed protocol, feed the analysis to a committee of statisticians and epidemiologists, and generate therapeutic guidelines. But, as he learns to his dismay, this is not enough, for rogue physicians persist in offering expensive and seemingly inappropriate remedies of their own. These physicians must therefore be motivated, bribed or otherwise encouraged to comply - for example, by changing the formulary. Come to think of it, the apparatchik now realises, a paramedic with a computer would be much less trouble. Problem solved? Not yet, alas, for patients, as he goes on to discover, are disinclined to forego their humble pleasures, are careless about taking their tablets and wantonly disregard his perception of their own best interests. Enter the educators, the psychologists, and all the other promoters of health. Some people will respond to this-for health promotion campaigns, like drugs for diabetes, work best when new-but many will not. Ah well, sighs the apparatchik, we did our best. And for those we failed to reach? Well, they can always go to their doctor.

There is, as the perceptive reader will appreciate, an element of satire in the above, but possibly not enough for comfort. The impact of the changing demography of diabetes will vary from one health system to another, and those who fear that humanity will soon be reduced to a homogenous admass should be encouraged by the extraordinary variation between national cultures when it comes to patient expectations and the delivery of diabetes care. The traditional brass plate still flourishes in some systems, and the physician who operates behind it will be content to see only those who want to see him, with no commitment to the rest. He will thrive in inverse proportion to the quality of primary care and in direct proportion to the magnitude of his fees. People value what is highly priced, a source of constant chagrin to those of us who work in the public sector, and typified for me by the patient who began the consultation by complaining of the cost of the bus ticket to the hospital. 'Look at it this way,' I suggested, 'in return for your bus fare you get to see a well-known diabetes specialist entirely free of charge.' The patient cheered up immediately. 'That's good,' he said, 'when do I get to see him?' Even those whose amour propre is not at risk now experience increasing tension between reimbursement systems that seek to impose cost-effectiveness and conformity and their own personal sense of a physician's role. The challenge is more clearly defined within more highly evolved and integrated (or, as some would say, socialised) health systems, such as that of the UK, but rising patient numbers and health costs spell change for all of us, everywhere.

How have physicians responded to this changing environment? Some specialists are blessed with well-funded services that cater to selected patients who are eager to attend. They will no doubt continue to tell us how easy it is to manage diabetes, provided we adopt their own particular pet therapy. Super-specialism is an option, but only works well if the system can identify the right people to refer. Some colleagues have gone out of the clinic to become standard bearers for diabetes care in their own community, often with remarkable success. They face two problems. The first is health service entropy, a law that dictates that anything that works well will either be reorganised or allowed to run downhill. The second problem is one of ownership, for the person who raises a standard soon discovers that so many other hands have reached out to grasp it that he or she is unable to move at all. Others again disappear into an administrator's office, emerging from time to time to tell the rest how their job should be done. Research is an attractive alternative, but part-timers find it hard to be competitive in the laboratory, and patient-based research is now so large-scale as to offer diminishing scope for personal initiative. A few brave souls venture into the alternative reality of qualitative research, but those who succeed in learning the language are rarely able to communicate the insights they have gained. Last but not least are the evidence-based evangelists, perpetually at odds with the Big Pharma groupies who proliferate at the other end of the ideological spectrum. The possibilities are endless, and if all else fails the golf course beckons. But where, amid so much displacement activity, do we discover the role of the diabetes specialist physician?

A diabetes physician has no unique skills. He cannot aspire to be a super-technician like the interventional cardiologist, or to become so clever that no-one else can understand him, like the neurologist or pure endocrinologist. He 
does not diagnose, requires few investigations, has few treatment options that only he can provide, and he cures noone. His one core role, which has never really changed, is to sit down with fellow human beings and help them to organise diabetes around their life, rather than life around the diabetes. To this encounter he brings considerable inherited and personal experience, acquired at the interface between affected people, their circumstances, and the vast body of lore and information that is available to help them. This, he firmly believes, can make a difference: so much so that he is likely to consider it one of the more rewarding aspects of his existence. He believes that some of the people he meets will emerge from the meeting feeling less isolated, with more pride, motivation and confidence, ready once again to take back control of their own lives.

All very well, as the outsider will observe, very uplifting, but how do you measure it? And here we come to the heart of the problem. In 1997 the human race experienced a collective twinge of anxiety when a computer called Deep Blue defeated the reigning chess champion, Garry Kasparov, two games to one, three draws. The diabetes equivalent of this contest has yet to take place, but the outcome cannot be in doubt. There are only 64 squares on a chess board, and Deep Blue's circuits are not yet ready to take on Mrs Gladys Smith in the diabetes clinic. And what logical device, however massive, could fail to experience the electronic equivalent of a nervous breakdown when required to come up with a rational therapy for type 2 diabetes? Humans can skip across gaps in logic or knowledge by ascertaining what most of those sitting around a table would like to believe, a method known as consensus. No computer could hope to compete with this.

You can, when all is said and done (and more is generally said than done), direct treatment to individuals or to populations. Populations are easy, for diabetes management can indeed be reduced to a set of general rules. These rules will on aggregate perform more efficiently than even the bestinformed of clinicians, and the simplest of computers can be taught to apply them. This time, the computer wins. And when the right systems are in place and the right advice has been given, all should now be well in the best of all possible worlds. But is it? Yes, because you have identified and treated people earlier, and thereby bought them a little time, which is all that medicine can ever do. No, because the condition still runs its inevitable course, and the great majority of people with diabetes are still in unsatisfactory control. And here we make our biggest mistake, concluding that what we need is better and more effective therapies for diabetes. We already have effective therapies. What we lack, and thank goodness for it, are better and more effective patients. If you do not believe me, consider the computer game known as The Sims. This game, which appeals to control freaks and adolescent girls, allows the player complete control over a community of totally compliant individuals. These eat, exercise and excrete in total conformity to the wishes of their controller. Who could doubt that $99 \%$ of people with diabetes would achieve their glucose targets within such a setting? I rest my case.

The diabetes physician, to conclude on a philosophical note, would not be needed in a world which lacked two things: free will and contingency. Contingency, in nontechnical terms, means the muddle of everyday life, the muddle that keeps you and your patients so occupied that you and they have no time or energy to spare for the prevention of other future messy situations. And now I must try to avoid two such messy situations myself. First, I am sorry that I have consistently referred to the physician as 'he'. Don't blame me, blame the English language, which lacks an androgynous personal pronoun. Please understand that 'he' embraces 'she' at all points in this discussion, except perhaps with reference to golf. Next, a word for the diabetes therapist who is not a physician. I will not apologise to you, for this is all your fault. It is your energy, skill and understanding of other people that has threatened to make me redundant. You taught me my limits, told me things about my patients that I would never have guessed, and the team approach you introduced has been the greatest advance in diabetes care in my generation. You have tolerated me for so long that you will now, I hope, forgive my focus on the most vulnerable member of your team, which is myself. People like me, as I am forced to conclude, have nothing to offer other than experience and expertise; and expertise, being unmeasurable, will always be undervalued. It ranks with health and happiness as something never fully appreciated until absent, as when you witness the damage that lack of expertise can inflict upon other people's lives. Physicians, and all those who treat diabetes by talking to people about it, see individuals. Individuals don't have outcomes. An individual is an outcome. If you can understand this, you are in the right speciality, and doing the right job. 